\title{
Two new dynamic devices / orthoses for training / assisting / rehabilitation of hand functionality in patients with stroke as a result of clinical experience
}

\section{DESIGN OF AN INTERVENTIONAL RANDOMIZED CASE-CONTROL STUDY TO TEST THEIR EFFECTIVENESS}

\author{
Catalin Moghioroiu, ${ }^{1,2}$ \\ ${ }^{1}$ University of Medicine and Pharmacy „Carol Davila” Bucuresti \\ ${ }^{2}$ National Clinical Center for Children Neuro-Psycho-Motor Rehabilitation „Dr. Nicolae Robanescu” \\ Bd Eroii Sanitari Nr. 8, Sector 2 /Str. Dumitru Minca, nr.44, Sector 4 - București catalinmoghi@yahoo.com
}

\begin{abstract}
Introduction - Observing stroke patients with hemiplegia we can easily identify one of the problems they face -inability to use affected upper limb. On a closer analysis we can see that this incapacity is determined, at many of them, by the incapacity to open the hand and grasp. They follow therapies for months and the results are often mediocre therefore respectively upper limb is neglected and forgotten and its activities are taken up by the healthy upper limb. These observations have prompted the search for other type of solutions to help / assist them and facilitate participation. In this way were created twodynamic devices/orthotics that assists finger extension, facilitates grasping and increase, at least in theory, the possibilities of participation.

Objective - The main purpose of this study is to test the effectiveness of using these devices on short and medium term.

Material and method - For this study we will select 60 patients with single stroke. Patients will be randomized into two groups. Patients in the control group will receive a physical therapy session per day for 10 days. Patients in the experimental group will receive in addition a functional workout (20-30 minutes of handling small objects and few simple functional gestures of the "activities of daily living") using one of the two devices the one that will allow the patient to best accomplish the given tasks. We will perform an initial assessment, one final and one to three months. The initiation of this study will be made after obtaining the opinion of the ethics committee, and the inclusion of patients will occur only after obtaining informed consent.

Conclusions - Creation of two dynamic devices/orthotics witch assist finger extension and facilitate prehension opened the possibility of carrying out a clinical trial to test the effectiveness of their use. To what extent that this will happen remains to be seen.
\end{abstract}

Keywords: stroke, hand rehabilitation, assistive dynamic orthotics / devices, functional training, participation.

\section{Introduction}

According to the WHO 15 million people worldwide suffer a stroke each year; about 5 million of them remain permanently disabled. In Europe stroke is the leading cause of morbidity and long term disability, according to the ESO (European Stroke Organization), estimating an increase in the yearly number of accidents from 1.1 million in 2000 to more than 1.5 million in 2025
(1). In the National Report regarding State of Health of Romania's Population from 2015 we find that the frequency of stroke is 3-4 times higher in Romania than in Western European countries (p. 34) and in Chapter Burden of disease estimated by $D A L Y$, where the burden of disease is measured by years of life adjusted for disability (disability adjusted life years) which is the sum of years of life lost by 
premature deaths and years lived with disability caused by the presence of disease or accidents, adjusted for severity of illness, we find that DALY per 100,000 population in 2012 is 3651 (p. 36) (2)

According to the ICF classification (3) the proper functioning of a person must be reflected in its participation in the activities of daily living (feeding, housekeeping, self-care, communication, recreation, etc.). This functional independence is the fundamental purpose of rehabilitation and it can be reduced essentially to walk and use of upper limb in daily activities. From this point of view "a poor outcome of the upper limb is considered a major threat to perceived quality of life in patients suffering from stroke (4)" and unfortunately "only one third of all stroke patients have regained some dexterity at 6 months "(5).

Between $2012 \quad-2014$, as an employee of University of Medicine and Pharmacy "Carol Davila" -Department of Rehabilitation at the National Institute of Physical Medicine Rehabilitation and Balneoclimatology (Filantropia), I evaluated a total number of approximately 100 adult patients with -ischemic or hemorrhagic stroke with left or right hemiplegia. Some of them came at few weeks after stroke, directly from neurology wards others later. Most patients benefited from two weeks of therapy and return after 6 months. Between hospitalizations some of them were taking physical therapy hours at home under the supervision of a physiotherapist and others were hospitalized for another two weeks in another hospital.

The patients enjoyed in the hospital of 2 sessions of physiotherapy a day (passive mobilization, auto passive, active, mechanical etc.) lasting about an hour plus physiotherapy procedures -electrotherapy, paraffin applications and possibly massage. For the most advanced of them (in terms of hand functionality) at physiotherapy sessions were used elements of occupational therapy -small objects manipulation (cubes, balls, discs, elements of an Canadian boards -zippers, laces switches, plugs, locks, door handles etc.) The aspects there were followed were: maintaining range of motion, decreasing muscle tone (if spasticity was present), increasing control, coordination and balance and cardio training. All of this in order to regain or improve gait and upper limb functionality. Physiotherapy sessions were held in a room of about 30 square meters with beds and equipment where worked in the same time10-15 people (patients, caregivers and therapists).

Most patients were assessed at admission and at discharge. In some cases, due to accumulated fatigue, the first evaluation was done on Tuesday (the second day of hospitalization), after patients getting used with the program, and recovered from the fatigue of the first day and the final assessment was done, in many cases, Thursday in week 2 before discharge (some patients prefer to go home Friday morning), after about 10 days of therapy.

For some of them, who returned after six months, it could also be done a new assessment at that time. The main aspects that were tracked and evaluated for the upper limb were: control and coordination, functionality, spasticity, pain and range of motion. For these aspects I used upper limb component of Fugl-Meyer Assessment (FMA) (7), a set of small pieces Ashworth scale, Visual Analog Scale (VAS) and a goniometer.

Fugl-Meyer is an excellent scale that assesses, among others, control and coordination of the upper and lower limb inside and outside synergies. "Items in the motor domain have been derived from Twitchell's 1951 description of the natural history of motor recovery following stroke and integrates Brunnstrom's stages of motor recovery" (Gladstone et al. 2002; Poole \& Whitney, 2001)( 7). Fugl-Meyer assesses movements of the shoulder, elbow, forearm, wrist and hand. 
I used the set of small pieces mainly to assess somehow functionality -the patient's possibility to manipulate these parts in a given time (where prehension was possible). Perhaps it would be more useful to use, for dexterity evaluation, a standardized test such as Nine Hole Peg Test or Box and Block Test. I did this taking in consideration that "Items of the FMA are intended to assess recovery within the context of the motor system" and "functional tasks are not incorporated into the evaluation" (Chae, Labatia, \& Yang, 2003) (7)"

Aschworth scale is perhaps the most known and used scale for spasticity evaluation, although both the evaluation method and the relationship between spasticity and disability after stroke begin to be questioned in recent years. $(8,9)$

For scientific rigor it would be required to process recorded data in a retrospective study, but until then I will mention a few aspects that I have noticed in practice, the conclusions I have drawn and solutions that have emerged.

When we evaluate and treat a hemiplegic patient we face at least three problems:

1. Loss of motor control simplistically defined as "the systematic transmission of nerve impulses from the motor cortex to the motor unit, resulting in coordinated muscle contractions." (12 pg. 69) by removing / disorganization of motor image from cortical level

2. the spasticity occurrence „velocity-dependent increase in muscle tone" according to an older definition (Lance, 1980) and

3. increase in the mechanical resistance of soft tissue mainly due to nonuse

It is therefore necessary to differentiate between "increased excitability of spinal alpha motor neurons and structural muscle changes that occur simultaneously" each benefiting from totally different evaluation and treatment modalities (11).

Although the encountered deficits were variable most patients regain walking pretty quickly (even if this does not guarantee that they had a good control of the lower limb (12 Pg. 718), I could not say the same thing about the hand functionality. In this regard it is worth mentioning that efficient prehension is the final and most important gesture of a functional hand, without ruling out the importance of the possibility to mobilize the elbow and the shoulder. Functionally speaking, an increased spasticity as much as lack of muscle tone may affect the prehension, yet some degree of spasticity is preferable to lack of muscle tone, because "disability resulting from motor neuron lesion may be due to poor motor control rather than spasticity" (Bakheit et al) (11).

From a clinical point of view the muscle tone determines the prehension. In this regard I met:

- patients with normal or slightly increased / decreased tone of forearm muscles and possible prehension

- patients with flaccid muscles and impossible prehension

- patients with very spastic muscles and eventually with muscle stiffness and impossible the prehension as well as

- patients who had an increased muscle tone that succumb to slow stretching that could grab an object in this way but do not manage to release it later.

The last ones drew my attention.

In addition I noticed that if the patient had a good result at the initial assessment that allowed him/her a better and faster recovery, but if the initial assessment result it was poor the subsequent results were also poor. From this point of view the correlation between the results obtained at the evaluation, the stroke moment and intervention moment is very important waiting, theoretically, better results in 
patients at whom intervention was made early.

For most patients I did not notice spectacular results (most of them had an increase of 2-3 points on the Fugl-Mayer scale in two weeks of therapy) nor within the same hospitalization nor from one admission to the next. Maybe do to the fact that majority of them were older or maybe the intervention was not done very early after stroke? Or maybe because of the methods used or maybe the actual working time on hand was limited? Only one patient had an increase of over 20 points in a year with an individual program at home of 6-8 hours a day, scheduled on hours with an hour of occupational therapy and 2 hours of daily household activities.

Very few patients were wearing prescribed rigid orthoses, either from convenience either because of discomfort.

Some patients had shoulder pain and stiffness aspects that affected the upper limb range of motion and functionality.

Some patients had a lower participation and weaker results at the end of the week, perhaps due to accumulated fatigue.

I noticed that in some patients at times when their mood improve also their participation and performance of the exercise improve.

Thinking at these aspects appeared some questions.

According to ICF classification participation and implicitly functionality are especially important (3)

Impairment of the upper limb in hemiplegia, especially if the affected limb is also the dominant one negatively influences participation. Improving functionality could also increase, at least in theory, participation.

The progress in the recovery process is slow and rather modest. Which are the factors that determine it? To what extent is determined by the initial recovery potential? There predictors of future evolution?
How important is the chosen method? There are more effective methods for upper limb rehabilitation with efficiency proven in clinical trials? If yes, then they should be known and used.

How important is hand splinting for the modulation of muscle tone? There are models of more efficient orthotics that facilitates compliance?

What can be done with patients who recovers very little or at all?

How important is the patient's mood in rehabilitation process and how can it be improved? Are other factors that influence participation?

There is an "optimal" amount of effort that potentiates the recovery process?

Are the quantity, quality and diversity of exercises important factors for progress?

How important is the context in which the patient works? How important is the moment when the patient is evaluated? It necessary to take into consideration the individual own biorhythm when we schedule physical therapy session?

\section{Present scientific literature answer to some extent at the questions above:}

- Regarding recovery potential and predictive factors one study (13) show that „accurate prediction of upper limb function is possible within 72 hours after stroke" the „patients with some finger extension and shoulder abduction on day 2 after stroke onset had a $98 \%$ probability of achieving some dexterity at 6 months", $60 \%$ of them „regained full recovery of upper limb function according to the ARAT (Action Research Arm Test) at 6 months”. The observations are analyzed and confirmed in other studies;

- $\quad$ Regarding used methods and their effectiveness in the rehabilitation of upper limb motor deficit secondary to stroke, a Canadian database (http://www.strokengine.ca) examines available clinical trials and make an evidence based synthetic analyze of their efficiency. The fallowing methods are take into consideration:

o Bilateral Arm Training 
o Functional Electrical Stimulation

o Mirror Therapy

o Motor Imagery / Mental Practice

o Repetitive Transcranial Magnetic Stimulation (rTMS)

o Task-Oriented Training

o Video Game Training

o Virtual Reality

o Constraint-Induced Movement

Therapy

- Regarding splinting efficiency an analysis from 2003 of studies published until then concludes that "There was insufficient evidence to either support or refute the effectiveness of hand splinting for adults following stroke", even so only 5 studies could be included (meet inclusion criteria) in this analysis of which only one of high quality (28 participants) (14)

- In terms of time allocated to exercise a meta-analysis found that, compared to control groups, an addition of at least 16 hours of training during the first six months after a stroke "has a small but positive effect on ADL" but without significantly influence dexterity (15). Another study concludes that „Greater intensity of leg rehabilitation improves functional recovery and health-related functional status, whereas greater intensity of arm rehabilitation results in small improvements in dexterity, providing further evidence that exercise therapy primarily induces treatment effects on the abilities at which training is specifically aimed" (16)

Analyzing the patients whom I evaluated and especially those who have enough strength to catch objects if someone/something open their hand but are no longer able to relax muscles to release the object and suspect that their condition could be maintained and even worsen, due to the fact that they do not have the possibility of a real recovery even with the help of physical therapy, I thought that a device that will assist hand opening could help them participate more and it would be also more efficiently.
Out of these observations came "The device for training / assistance / rehabilitation of hand functionality at the patient with sequelae after stroke or other central motor neuron lesions." Device for which was filed a patent application at OSIM (STATE OFFICE FOR INVENTIONS AND TRADEMARKS), the application was published in BOPI (Official Industrial Property Bulletin) No. 5 in 2014 (10).

The technical problem solved by the device proposed for certification is that manages to transform hemiplegic patient hand with spasticity at the upper limb level in an effective grip, allowing him to participate in functional activities whose benefits are often highlighted in the literature. The device accomplishes this by assisting finger extension (using variable elastic resistance chosen depending on the degree of spasticity of the patient), open the spastic fist and creating a cavity that allows gripping and moving small objects. Fingers and palm taking the external contour shape of a sphere.

In an attempt to find simple and more functional solutions for patients, analyzing some products on the market I created also "The dynamic orthosis for training / assistance / rehabilitation of hand functionality at the patient with sequelae after stroke or other central motor neuron lesions." Device for which was filed a patent application at OSIM, the application was published in the same BOPI No. 5 in 2014 (10).

The technical problem solved by the orthosis proposed for certification is that manages to transform the small move (especially fist extension) that is present even at the most affected patients (with hemiplegia secondary to stroke), in a functional exercise by releasing the wrist and positioning it in flexion, by assisting extension of fingers two and three and thumb abduction, allowing the first tree fingers to grip with minimum of effort and 
giving the patient the opportunity to participate in therapies that generates results and motivation, increasing compliance and thus effectiveness of therapy.

In order to understand this problem are useful a few concepts of functional anatomy: at the tendons level of flexors and extensors of the wrist and fingers there is an interdependence so that wrist flexion facilitates fingers extension and wrist extension facilitates fingers flexion. Disturbing this balance is even more important at hemiplegic patients with spasticity on the flexor muscles of the wrist and fingers to whom in addition occurs the tendons shortening of deep and superficial flexor muscle of the fingers, so that wrist extension further contributes to stronger flexion of the fingers. Although it is desirable to attempt through posture and stretching to maintain a muscular tone and length closer to normal, however, to hope for some degree of extension of the fingers and therefore of hand functionality should that, in such cases, the fist remain slightly flexed, in this way facilitating participation in functional activities so useful. Another thing that has to be known is that hand functionality is given in the highest percentage by the thumb functionality and the clamp made by the first three fingers this thing is even more evident to the patients where the affected hand is the dominant hand. An interesting thing that can be seen in some hemiplegic patients is that quite early they get fist extension unfortunately simultaneously with strong fingers flexion, the described orthosis manages to make usable this movement namely enables patients to achieve prehension using fist extension. Beyond training opportunities that it offers this orthosis, with its help, the patient can more easily participate in everyday activity, for example can grasp a door handle and, at least theoretically, can open the door.

Taking into account the achieved experience, the data from current scientific literature and the possibility of using the two dynamic devices/ orthotics I believe it is proper to initiate an interventional, casecontrol, randomized, clinical trial, study that would have as main goal:

- to test the effectiveness of using the two devices and show if these are able to increase immediately, but also on longterm, the hand functionality, and if so which patients may benefit most from their use, and then

- to show to what extent the (relatively) intense workout with the two devices manages to produce adaptive changes (muscular and neural) needed to growth hand functionality in their absence.

For this study we will go through the following steps:

1. Identification of a clinic willing to allow the conduct of this study within it.

2. Obtaining the opinion of the ethics committee.

3. Notification of National Agency of Medicines and Medical Devices to the extent that this is necessary in accordance with the the legislation in force and the requirements of the ethics committee.

4. Obtaining informed consent from patients.

5. Initiation of the study.

6. Data collection

7. Data analysis

8. Interpretation of results

9. Drawing conclusions

10. Writing a new article

\section{Material and method}

For this study we will select a total of 60 patients with a single stroke, CT/MRI diagnosed, that will meet the following inclusion / exclusion criteria:

- A single stroke

hemorrhagic/ischemic stroke CT/MRI diagnosed

- $\quad$ The intervention (physical therapy start) has to be done in the first year after stroke preferably in the first 3 months

- $\quad$ Maximum age 70 years 
- $\quad$ Signing of informed consent and the consent of filming

- $\quad$ A deficit of affected upper limb of at least 10 points on Fugl -Mayer Assessment - Capacity to understanding and cooperation, lack of attention deficit

- $\quad$ Possibility of seating and standing unsustain at least few minutes

- Possibility of autonomous movement and exercise tolerance

- $\quad$ Ability to perform given exercises at least some degree of shoulder and elbow control

\section{- $\quad$ Absence of vision problems}

- Absence of serious associated illnesses

Patients will be randomized into two groups. Patients in the control group will receive a daily physical therapy session for 10 days. Patients in the experimental group will receive in addition a functional training session each day (ADL and handling small objects) using one of the two devices the one that will enable the patient to best accomplish given tasks. Considering the fact that, if there is a force deficit, the device with a circle shape has a greater potential to train it. Functional training session will be preceded by a 5-10 minutes period of accommodation in which it will be carried out passive, active or self-passive mobilizations. The session will last about 20-30 minutes depending on the tolerance of each individual. We will try to work in teams of two patients to bring extra motivation and facilitate participation.

We will perform an initial assessment (at admission) and a final one (at discharge) and one at three months. We will assess: control and coordination at upper limb level using Fugl-Meyer Assessment, range of motion on the basic movements of the shoulder, elbow, wrist and hand, pain with VAS and spasticity of the muscles of the shoulder, elbow and hand with Ashworth scale. We will test their effort capacity to see to what extent physical condition affects upper limb performance. We will try to find a way to assess the mood to see to what extent the mood influences the moment performance. We will evaluate the dexterity and we will test a number of ADL. It will be noted the number of exercises sets that the patient can achieve in one session. To these evaluations we will add the filming.

\section{Conclusions}

The increasing incidence of stroke, its appearance at professionally active people, the disabling deficit that leave behind for a long time from the day of onset, the deficit that is not resolved by spontaneous recovery that occurs in the first weeks on one hand and on the other hand the long duration of therapy, the high cost and scarcity of trained physical therapists to conduct high quality therapy in accordance with the current findings of neurorehabilitation makes necessary continues research to develop therapies that are deep scientifically based and increasingly more efficient. The only justified criterion in their evaluation being that of their effectiveness -to what extent they restore functionality and generate participation, criterion that it is centered on the patient with its needs and desires.

The accumulated experience in rehabilitation of patients with hemiplegia secondary to stroke led to creation of two dynamic devices / orthoses that have the theoretical potential to increase the functional performances to some of the stroke patients and to improve their participation. The clinical study that we propose for deployment aims to demonstrate to what extent this can be done.

\section{Bibliografie}

1. www.eso-stroke.org 
2. http://insp.gov.ro/sites/cnepss/wpcontent/uploads/2014/11/ECHI.pdf

3. http://www.who.int/classifications/dr afticfpracticalmanual2.pdf?ua=1

4. Nakayama H, Jørgensen HS, Raaschou $\mathrm{HO}$, Olsen TS: Compensation in recovery of upper extremity function after stroke: the Copenhagen Stroke Study. Arch Phys Med Rehabil 1994, 75:852-7. (Citat in 6)

5. Dobkin BH: Clinical practice. Rehabilitation after stroke. $N$ Engl $J$ Med 2005, 352:1677-84. (Citat in 6)

6. Gert Kwakkel*1,4, Carel GM Meskers2, Erwin E van Wegen1, Guus J Lankhorst1, Alexander CH Geurts3, Annet A van Kuijk3, Eline Lindeman4, Anne VisserMeily4,5, Erwin de Vlugt6 and J Hans Arendzen Impact of early applied upper limb stimulation: The EXPLICIT-stroke programme design 2December 2008 BMC Neurology 2008, 8:49 doi:10.1186/1471-23778-49

(http://bmcneurol.biomedcentral.com/articles/1 0.1186/1471-2377-8-49)

7. http://www.strokengine.ca/family/fma family/

8. Lindberg $P G$, Gäverth J, Islam $M$, Fagergren A, Borg J, Forssberg H. Validation of a new biomechanical model to measure muscle tone in spastic muscles. Neurorehabil Neural Repair 2011, Apr 13

9. Spasticity After Stroke Its Occurrence and Association With Motor Impairments and Activity Limitations Disa K. Sommerfeld, PT, MSc; Elsy U.-B. Eek, PT, MSc; Anna-Karin Svensson, PT, MSc; Lotta Widén Holmqvist, PT, PhD; Magnus $\mathrm{H}$. von Arbin, MD, PhD (Stroke. 2004;35:134-140.) http://stroke.ahajournals.org/content/35/1/13 4.long

10. Official Industrial Property Bulletin BOPI No. 5 in 2014 http://www.osim.ro/publicatii/brevete/bopi 201 4/bopi_inv 05 2014.pdf

11. Påvel G. Lindberg, PhD1, 2, Marc A. Maier, PhD2, 3, Jörgen Borg, PhD1* si Anthony Ward, BSc, MD, FRCPEd, FRCP Commentaries on "SPASTICITY OR REVERSIBLE MUSCLE HYPERTONIA?” J Rehabil Med 2011; 43: 812-815 si

RESPONSE TO LINDBERG ET AL.'S AND WARD'S COMMENTARIES Abdel Magid O. Bakheit, MD, PhD1, Klemens Fheodoroff, MD2 and Franco Molteni, MD3
12. Darcy Ann Umphred, Rolando Lazaro, Margaret Roller, Gordon Burton Neurological Rehabilitation, 6th Edition Ed. Elsevier 2013

13. Presence of Finger Extension and Shoulder Abduction Within 72 Hours After Stroke Predicts Functional Recovery Early Prediction of Functional Outcome After Stroke: The EPOS Cohort Study Rinske H.M. Nijland, MSc; Erwin E.H. van Wegen, PhD; Barbara C. Harmeling-van der Wel; Gert Kwakkel, PhD; on behalf of the EPOS Investigators Stroke. 2010;41:745-750

http://stroke.ahajournals.org/content/41/4/745

14. NA Lannin, RD Herbert Is hand splinting effective for adults following stroke? A systematic review and methodological critique of published research - Clinical Rehabilitation, 2003 cre.sagepub.com

15. Kwakkel G, van Peppen R, Wagenaar RC, Wood Dauphinee S, Richards C, Ashburn A, Miller K, Lincoln N, Partridge C, Wellwood I, Langhorne P: Effects of augmented exercise therapy time after stroke: a meta-analysis. Stroke 2004, 5:2529-39. http://stroke.ahajournals.org/content/35/11/2529 16. Kwakkel G ${ }^{1}$, Wagenaar RC, Twisk JW, Lankhorst GJ, Koetsier JC.Intensity of leg and arm training after primary middle-cerebralartery stroke: a randomised trial. Lancet. 1999 Jul 17;354(9174):191-6. (abstract on https://www.ncbi.nlm.nih.gov/pubmed/1042130 0) 